\title{
Os desafios durante a campanha de vacinação contra COVID-19: um relato de experiência e reflexões
}

\author{
Challenges during the vaccination campaign against COVID-19: an experience report and \\ reflections
}

Retos durante la campaña de vacunación contra COVID-19: relato de experiencia y reflexiones

Douglas do Nascimento Galvão ORCID: https://orcid.org/0000-0001-5277-0718 Universidade Federal do Pará, Brasil E-mail: douglasnascimentogalvao@gmail.com

Elian Coimbra Fontinelli Tavares ORCID: https://orcid.org/0000-0003-2886-935X Universidade Federal do Pará, Brasil E-mail: judacoimbra@gmail.com

Leonardo Carvalho da Silva ORCID: https://orcid.org/0000-0001-8554-2391 Universidade Federal do Pará, Brasil E-mail: leonardohab2020@gmail.com

Vitória Yasmin Sousa Correia ORCID: https://orcid.org/0000-0001-7307-3508 Centro Universitário FIBRA, Brasil E-mail: vitoria131correia@gmail.com Jéssika Sayuri Campelo Kato

ORCID: https://orcid.org/0000-0003-3114-0594 Universidade do Estado do Pará, Brasil E-mail: jessika.kato@aluno.uepa.br

Lucas Matheus da Silva Castro

ORCID: https://orcid.org/0000-0003-3570-8669 Universidade do Estado do Pará, Brasil E-mail: lucas.mcastro@aluno.uepa.br

Paula Gabriela Nascimento Gonçalves

ORCID: https://orcid.org/0000-0002-1292-0715 Universidade do Estado do Pará, Brasil E-mail: paula.goncalves@aluno.uepa.br

Arley Guilherme Santos de Sousa ORCID: https://orcid.org/0000-0003-0717-8541 Universidade Federal do Pará, Brasil E-mail: arley.sousa@ics.ufpa.br

Mainã Cristina Santos dos Santos ORCID: https://orcid.org/0000-0002-4246-0078 Universidade do Estado do Pará-, Brasil

E-mail: mcsantosdossantos@hotmail.com

Moisés Felipe Silva da Conceição ORCID: https://orcid.org/0000-0001-9123-9612 Universidade Federal do Pará, Brasil E-mail: moisesfelipe.uepa@gmail.com

Eduarda Beatriz de Azevedo Silva ORCID: https://orcid.org/0000-0002-0819-8299 Faculdade Maurício de Nassau, Brasil E-mail: eduardabeaazevedo@gmail.com

Wellen Sampaio Ferreira

ORCID: https://orcid.org/0000-0002-1317-4339 Universidade Federal do Pará, Brasil E-mail: wellen.ferreira@ aluno.uepa.br Albertth Alex da Silva Lima ORCID: https://orcid.org/0000-0003-4309-8805 Universidade Federal do Pará, Brasil E-mail: albertth.lime10@gmail.com

Carlos Arthur da Silva Milhomem ORCID: https://orcid.org/0000-0001-8020-1088 Universidade do Estado do Pará, Brasil

E-mail: carlos.milhomem@aluno.uepa.br 
Dandara de Fátima Ribeiro Bendelaque

ORCID: https://orcid.org/0000-0002-5580-284X

Universidade Federal do Pará, Brasil

E-mail: bendelaqued@gmail.com

\begin{abstract}
Resumo
Objetivo: Analisar e descrever a experiência e desafios dos voluntários, durante a campanha de imunização contra a COVID-19, em um posto de vacinação, localizado na Região metropolitana de Belém. Metodologia: Trata-se de um estudo descritivo, com abordagem qualitativa, do tipo relato de experiência, realizado por acadêmicos e profissionais voluntários de diversos cursos, com prevalência da área da saúde, durante a campanha de vacinação contra a COVID19, no município de Belém-PA, no período de Fevereiro a Abril de 2021. Resultado: Realizada em ambiente extramuros, a campanha de vacinação contra Covid-19 deparou-se com vários desafios para a sua realização, entre eles: a organização logística do espaço físico para o condicionamento das pessoas, o acolhimento e sua humanização, assim como, suas subdivisões e a interação bilateral entre profissionais e os futuros vacinados e seus familiares. Conclusão: Verifica-se a necessidade de um maior aprofundamento sobre a temática com a adoção de novas pesquisas sobre o atual cenário na tentativa de minimizar os problemas encontrados e consequentemente agilizar e colaborar com o processo de adesão da vacinação pela população.
\end{abstract}

Palavras-chave: Vacinação; COVID-19; Idosos.

\begin{abstract}
Objective: Analyze and disclose the experience and challenges of two volunteers, during the immunization campaign against COVID-19, at a vaccination post, located in the Metropolitan Region of Belém. Methodology: This is a descriptive study, with a qualitative approach, of the experience report type, carried out by academics and volunteer professionals from different courses, with prevalence in the health area, during the vaccination campaign against COVID-19, in the city of Belém -PA, from February to April 2021. Result: Carried out in an extramural environment, the vaccination campaign against Covid-19 faced several challenges for its realization, among them: the logistic organization of the physical space for the conditioning of people, the reception and its humanization, as well as its subdivisions and bilateral interaction between professionals and future vaccinated people and their families. Conclusion: There is a need for greater depth on the subject with the adoption of new research on the current scenario in an attempt to minimize the problems encountered and, consequently, streamline and collaborate with the process of adherence to vaccination by the population.
\end{abstract}

Keywords: Vaccination; COVID-19; Aged.

\title{
Resumen
}

Objetivo: Analizar y describir la experiencia y desafíos de los voluntarios durante la campaña de inmunización contra COVID-19, en un puesto de vacunación, ubicado en la región metropolitana de Belém. Metodología: Se trata de un estudio descriptivo, con enfoque cualitativo, de la experiencia. tipo de informe, realizado por académicos y profesionales voluntarios de diversos cursos, con prevalencia en el área de salud, durante la campaña de vacunación contra COVID19, en la ciudad de Belém-PA, de febrero a abril de 2021. Resultado: Realizado en un ambiente extramuros, la campaña de vacunación contra Covid-19 enfrentó varios desafíos para su realización, entre ellos: la organización logística del espacio físico para el acondicionamiento de las personas, la recepción y su humanización, así como, sus subdivisiones y la interacción bilateral entre profesionales y los futuros vacunados y sus familias. Conclusión: Es necesario profundizar en el tema con la adopción de nuevas investigaciones sobre el escenario actual en un intento de minimizar los problemas encontrados y, en consecuencia, agilizar y colaborar con el proceso de adherencia a la vacunación por parte de la población.

Palabras clave: Vacunación; COVID-19; Anciano.

\section{Introdução}

O novo Coronavírus, denominado de SARS-CoV-2, foi identificado pela primeira vez em 31 de dezembro de 2019 na cidade de Wuhan, na China, sendo integrante de uma família de 7 variantes do coronavírus causadores de infecções respiratórias sazonais. Configura-se como uma doença de elevada transmissibilidade e com distribuição global, responsável por ocasionar uma infecção respiratória aguda potencialmente grave à evolução clínica do paciente e com rápida disseminação, tomando, dessa forma, dimensões continentais. Sua transmissão ocorre principalmente por meio de gotículas respiratórias e/ou pelo contato com superfícies contaminadas (Brasil, 2020).

Em virtude do elevado nível de transmissão, passou a ser classificada como pandêmica, mudando drasticamente a forma de combate ao vírus. Desde então, tornou-se imprescindível a adoção de protocolos, procedimentos e guias na tentativa de 
identificar e conter o avanço do vírus e da doença em seu estado mais grave, através das recomendações de uso de máscaras, a utilização de álcool em gel, a lavagem das mãos, o distanciamento social e o decreto de quarentenas se tornaram parte do cotidiano das pessoas (OMS, 2019).

O contato com uma variante desconhecida e a sua proliferação exacerbada, gerou o colapso dos Sistemas de Saúde no Mundo, cerca de $40 \%$ das pessoas contaminadas desenvolvem a forma leve ou moderada da doença e $15 \%$ delas desenvolvem a forma severa, necessitando de suporte de oxigênio. Outras 5\% das pessoas desenvolvem a forma grave e podem apresentar quadros de complicações respiratórias, complicações sistêmicas como trombose, complicações cardíacas, sepse e outras. Diante disso, tornou-se urgente a busca por uma vacina que auxiliasse no processo de diminuição de casos graves, internações e mortes, dando início ao desenvolvimento de um imunobiológico que fosse eficaz e seguro na mitigação dos impactos da pandemia (Brasil, 2020; Atehortua \& Patino, 2021).

No Brasil, os estudos empreendidos pelo Instituto Butantan em associação com a empresa farmacêutica Chinesa Sinovac e pela Astrazeneca/Universidade de Oxford em associação com a Fundação Oswaldo Cruz, proporcionou ao país no dia 17 de janeiro de 2021 a aprovação dos seus dois primeiros imunizantes autorizados pela ANVISA para uso nacional (Brasil, 2020).

O estado do Pará apresentou o Plano Paraense de Vacinação (PPV-Covid-19), no qual descreveu as estratégias e ações de operacionalização da vacinação contra a COVID-19 nos 144 municípios do estado, em conformidade com os protocolos de vacinação de nível federal. Dessa forma, o planejamento estabeleceu os grupos prioritários para vacinação - com a meta de alcançar 95\% de cobertura vacinal de cada grupo-alvo - como serão feitas a comunicação de informações pertinentes para a população-alvo e planejamento logísticos de recursos existentes necessários para a campanha (SESPA, 2021).

Foram priorizados grupos que apresentam maior risco de hospitalizações e óbitos por Covid-19, dentre eles, destacamse as pessoas com mais de 60 anos, que apresentam o dobro de risco comparado com os demais casos, sendo que essas taxas aumentam conforme a idade, chegando a 8,5 para hospitalização e 18,3 para óbito entre idosos com 90 anos ou mais. (Brasil, 2021)

Desde o início da campanha, foram divulgadas diferentes informações que repercutiram de maneira negativa, influenciando diretamente na adesão dos idosos e seus familiares à vacina. Diante disso, os voluntários encontraram desafios durante a campanha. Dessa forma, esse estudo tem como objetivo analisar e descrever a experiência e desafios dos voluntários, durante a campanha de imunização contra a COVID-19, em um posto de vacinação, localizado na Região metropolitana de Belém.

\section{Metodologia}

Trata-se de um estudo descritivo, com abordagem qualitativa, do tipo relato de experiência, realizado por acadêmicos e profissionais voluntários de diversos cursos, com prevalência da área da saúde, durante a campanha de vacinação contra a COVID-19, no município de Belém-PA.

O estudo descritivo teve como objetivo determinar a distribuição de doenças ou condições relacionadas à saúde, segundo o tempo, o lugar e/ou as características dos indivíduos (Mendes, Silveira \& Galvão, 2019).

Em relação à pesquisa com abordagem qualitativa, sua escolha ocorreu devido a mensuração de resultados que não podem ser representados de forma estatística, realizando abordagem categórica dos resultados, com o aprofundamento da compreensão de um grupo social, de uma organização, etc (Minayo, 2012).

Segundo Cavalcante e Lima (2012), a metodologia relato de experiência tem como objetivo permitir a reflexão das ações realizadas de forma individual e/ou coletiva, sobre a realidade na qual foi vivenciada e, posteriormente, explanadas de forma descritiva para a comunidade acadêmica. 
A experiência ocorreu no período de fevereiro a abril de 2021, pelos discentes e profissionais voluntários que atuaram nas diferentes funções de vacinador, aspirador, apoio e registro. A Campanha de vacinação foi realizada a partir das logísticas e estratégias criadas pela Secretaria Municipal de Saúde (SESMA) em conjunto com o Ministério da Saúde, a fim de estabelecer metas e planos para o calendário vacinal da população.

A vacinação foi dividida conforme a faixa etária, sendo assim, a primeira fase da campanha com os profissionais de saúde no período de 21 de janeiro a 01 de fevereiro, em seguida vacinando os idosos com mais de 80 anos e acima de 60 anos que vivem em lares de longa permanência do dia 02 de fevereiro a 04 de março, e teve como meta vacinar, aproximadamente, 10 mil idosos que nasceram nos anos de 1939, 1940 e 1941 ou que completarão as respectivas idades até o mês de dezembro de 2021.

Na segunda fase, foi iniciada a vacinação dos idosos acima de 75 anos e moradores de rua, no período de 05 a 08 março. Já os idosos acima de 60 anos foram vacinados no período de 28 de março a 22 de abril, sendo definidas as datas separadamente para a primeira e segunda dose. A principal meta do plano vacinal era vacinar cerca de $95 \%$ da população.

Nesse cenário, foram estabelecidos cerca de 20 postos de vacinação do município de Belém, em universidades, igrejas, estádios, entre outros, em alguns postos foram fornecidos os atendimentos por meio do drive thru e vacinação a domicílio, para os idosos que apresentavam alguma dificuldade de locomoção.

As atividades foram planejadas conforme a necessidade e especificidade de cada posto, onde cada profissional ou voluntário tinha funções. O registro realizava a solicitação de documentos, anamneses e os intervalos entre as doses, já o vacinador tinha como principal função a verificação da carteira de vacina, cumprir com as normas de administração e conservação e orientar sobre os possíveis eventos adversos, por fim o apoio era responsável por organizar a fila, conforme as normas da vigilância, sempre mantendo as medidas de prevenção da COVID-19.

Os postos de vacinação eram compostos e coordenados por funcionários da Secretaria de saúde, acadêmicos de diversos cursos e instituições e residentes de diferentes áreas, os quais ocupavam as funções de vacinador, aspirador, apoio e registro. Destacamos que por se tratar de um relato de experiência, desempenhado a partir das vivências de diferentes sujeitos, não houve necessidade de submissão ao Comitê de Ética em Pesquisa.

\section{Resultados e Discussão}

Realizada em ambiente extramuros, a campanha de vacinação contra Covid-19 deparou-se com vários desafios para a sua realização, entre eles: a organização logística do espaço físico para o condicionamento das pessoas, o acolhimento e sua humanização, assim como, suas subdivisões que serão esclarecidas no decorrer do estudo e a interação bilateral entre profissionais e os futuros vacinados e seus familiares.

As salas de vacinas são consideradas áreas semicríticas e devem seguir rigorosos procedimentos ambientais com instalações adequadas para o seu funcionamento. Recomenda-se uma área média a partir de $9 \mathrm{~m} 2$ para a adequada disposição dos equipamentos e dos mobiliários além do fluxo de movimentação em condições ideais para a realização das atividades (BRASIL, 2014).

No entanto, haja vista o aspecto emergencial da vacinação e a deficiência de locais adequados para a realização da mesma, apareceram impasses que comprometem a difusão dos conhecimentos técnicos e padrões. Constatou-se que uma das principais dificuldades era o provimento de um lugar adequado para a acomodação da população de forma que evitasse o surgimento de aglomerações e promovesse a equidade no acesso.

Em um local de vacinação, é essencial que todos os procedimentos desenvolvidos promovam a máxima segurança, reduzindo o risco de contaminação para os indivíduos vacinados e também para a equipe de vacinação. Assim, adotou-se protocolos de boas práticas de imunizações que atendessem as especificidades básicas do manual de procedimentos para 
vacinação, onde o ambiente instável demandou a utilização de caixas térmicas para conservação e preservação dos imunobiológicos (Brasil, 2014; Santos, 2017).

Assim, de forma a evitar infecções cruzadas pela COVID-19, o espaço foi organizado dispondo de diferentes entradas e com o uso de cadeiras pela arquibancada de modo que mantivesse o distanciamento mínimo necessário entre as pessoas. $\mathrm{O}$ estabelecimento de tendas para o drive thru e a entrada exclusiva para essa modalidade, também permitiu que houvesse uma separação dos grupos presentes e a manutenção do distanciamento social. A acessibilidade e disponibilização de salas exclusivas para pacientes com necessidades especiais e grupos prioritários, inclusive com a disponibilização de cadeira de rodas para pacientes com dificuldades de locomoção, permitiu que a equidade fosse mantida.

Diante do exposto, foi necessária a adoção de protocolos internos como um meio de estabelecer uma homogeneidade no atendimento, logo sua agilidade. Tendo o tempo de espera dos pacientes para se vacinarem o principal entrave, pois os idosos deslocam-se para os postos de vacinação com até 5 horas de antecipação a abertura dos portões com receio de não haver vacinas suficientes para todos, gerando um cansaço físico e mental que se externaliza com impaciência frente aos profissionais que irão atendê-los, o que dificulta a criação da confiabilidade entre os participantes.

Esta situação também refletiu no atendimento por parte dos voluntários que viam-se constantemente expostos a conflitos e desafios relacionados ao contato direto com o público, enfrentando-os com o objetivo de zelar e preservar o exercício da sua atividade de forma segura, e esta exposição é um dos principais fatores desencadeantes da Síndrome de Burnout.

A Síndrome de Burnout (SB), também conhecida como síndrome do esgotamento profissional e psíquico, configura-se como uma doença de ordem psicossocial que surge como resposta aos estressores interpessoais de natureza crônica relacionados ao meio laboral, sendo um fenômeno que afeta profissionais que possuem intenso contato com os usuários de seus serviços, como os profissionais da saúde (Perniciotti, 2020; Ribeiro, 2020).

Quanto à logística e distribuição da rede de frios, torna-se um desafio assegurar a quantidade suficiente de imunobiológicos que serão administrados, a fim de evitar falta ou desperdícios de doses, bem como a garantia dos insumos necessários para a sua preservação e administração. Os imunobiológicos necessitam de condições adequadas de forma que mantenham suas características iniciais, a fim de conferir imunidade, já que configuram-se como produtos termolábeis e deterioram-se a partir de exposição prolongada a variações de temperatura (Santos, 2017).

Foi observada que a vacinação extramuro ficou à mercê das mudanças climáticas, além de outros aspectos que desafiavam a logística e manutenção da vacina. No entanto, como forma de minimizar possíveis impactos, utilizou-se caixas térmicas dentro das normas preconizadas pelo manual de vacinação para a conservação dos imunobiológicos, permitindo que as vacinas chegassem com agilidade e dentro dos parâmetros necessários para a imunização. Além disso, a utilização de tendas no sistema drive thru, e a cobertura artificial proporcionada pelo prédio, permitiu que houvesse uma manutenção da temperatura e proteção das vacinas e usuários contra as mudanças climáticas comuns à região.

Outro desafio analisado, foi o acolhimento dos usuários, realizado desde a chegada da pessoa a ser vacinada e seus familiares, que passam pelo inicialmente pelo registo até se vacinarem. Ele pode ser dividido em três momentos: registro, triagem e orientações, sendo importante a realização de forma simultânea e transversal dos mesmos. Tornando o registro uma ferramenta fundamental na análise do desempenho da campanha de vacinação, haja vista que os dados pessoais dos vacinados são utilizados na avaliação quantitativa dos grupos prioritários. (Sato, 2018)

Nesse contexto, foram encontradas certas resistências pelos acadêmicos, uma delas é a forma manual de realizar o registro em uma planilha impressa. Embora, essa tarefa esteja sendo realizado de maneira ágil e correta com o objetivo de manter o fluxo de pessoas e de carros - no caso do drive-thru - ainda sim, tem-se o risco de cometer erros no momento da anotação, podendo gerar informações errôneas para o sistema e dificultando na acurácia desses dados. (Brasil, 2021) 
Esse desafio é ainda mais evidente na modalidade drive-thru, no qual muitas vezes, os voluntários precisam realizar esse procedimento em paralelo aos carros, que por sua vez, estão em movimento. Ainda se tratando do registro, foi iniciada a sua realização por meio de um aplicativo para smartphones que sintetiza este processo, o qual tem o objetivo de facilitar a identificação do cidadão. Para isso, os registradores usam apenas o número de Cadastro de Pessoa Física (CPF) e, automaticamente, são exibidos diferentes dados sobre o indivíduo.

Observou-se a existência da problemática relacionada à demora do registro de informações pela secretaria de saúde do estado, quando se é feita pelo material impresso, assim, resultando na dificuldade de exibir esse elemento no aplicativo e aumentando o tempo de atendimento do usuário. Além disso, a ferramenta apresentou falhas de funcionamento, dentre elas, os erros no momento da busca pelo CPF e interrupções por exceder o limite de usuários simultâneos, assim como a dificuldade de acesso à internet.

O próximo ponto a ser destacado é a triagem, no qual era realizada a anamnese para identificar se há algum fator que impeça a pessoa de se vacinar, o que ocorre em casos de pacientes com febre, e outros sintomas do COVID, doença aguda e início agudo de doenças crônicas, vacinação recente contra influenza e H1N1. Também, é avaliado se houve reação anafilática em casos de segunda dose ou se há alergia a qualquer um dos componentes da vacina, com o objetivo de evitar Eventos Adversos Pós Vacinação (EAPV) (Brasil, 2020; Brasil, 2021).

É importante destacar que não foram disponibilizadas ferramentas necessárias para essa triagem, como oxímetro ou termômetro infravermelho. Ainda assim, devido ao conhecimento prévio, comunicação acessível e humanizada foi possível identificar esses pontos. Entretanto, constantemente, foram relatados situações em que usuários na fila de vacinação omitiram informações para que fosse autorizados a receber a dose, demonstrando descontentamento quando a aplicação do imunobiológico era negado temporariamente, decorrendo-se de forma reverente ou indelicada, o que exigiu dos voluntários uma nova abordagem para que fosse compreendido a importância desses procedimentos.

O último ponto a ser destacado do acolhimento são as orientações e interação que fazem parte do processo de humanização, no qual é possível entender a condição socioeconômica e o grau de exposição de informações que o cliente do sistema de saúde estar. A partir disso, os acadêmicos que estão na campanha se colocaram à disposição para esclarecer questionamentos acerca do processo de vacinação e da vacina propriamente dita, o que interfere significativamente na adesão dos usuários ao sistema, (Hamada et al. 2018)

Os acadêmicos tiveram a oportunidade de consolidar durante a prática exercida no posto, conceitos já debatidos em meio as aulas, como a exemplo da educação em saúde, processo de imunização, vacinação, e desenvolver habilidades interpessoais importantes para assistência (Montezeli \& Haddad, 2016).

Isso ocorreu, por intermédio de orientações de forma compreensível e sucinta para o público em que está sendo vacinado, orientando-os sobre cuidados que devem ser tomadas a partir da aplicação, também são destacados o percentual de eficácia, como a vacina induz o sistema imunológico a se proteger contra a doença e possíveis reações adversas, tornando os usuários mais confiantes, tanto em relação ao imunizante, quanto aos procedimentos utilizados, como aspiração da vacina e técnicas de aplicação do imunobiológico. (Vilanova, 2020)

Outro aspecto que se evidenciou neste estudo foram os meios de comunicação de massa, os quais começaram a noticiar doses sendo desperdiçadas, erros na aplicação de imunizantes, pessoas sem prioridade no momento passando à frente de outros grupos prioritários e ações judiciais que começaram a ser instauradas. Ademais, o fenômeno das informações falsas popularmente chamadas de fake news - criadas e difundidas nas redes sociais, principalmente em aplicativos de mensagens instantâneas, são propagadas com facilidade. Causando, assim, na população um certo alarme. (Atehortua \& Patino, 2021)

Por conseguinte, foram observadas inúmeras dúvidas que se relacionavam às informações falsas e também a grande exposição de informações fragmentadas. Dentre elas: dúvidas sobre as vacinas Coronavac do Instituto Butantan e Astrazeneca 
de Oxford, se tratando da eficácia da mesma, e dos possíveis efeitos adversos, respectivamente, da necessidade de se fazer a aspiração dentro das caixas térmicas e a confirmação de que o imunizante foi realmente aplicado, entre outras.

Além da preocupação com a correta realização da técnica, foi necessário o estabelecimento de comunicação assertiva entre os profissionais e os vacinados e seus acompanhantes, onde se pudesse alcançar o objetivo de vacinar os clientes e informálos de maneira correta, para que o ciclo vicioso das fake news fosse quebrado.

Foi observada a resistência em relação à aceitação da verdadeira informação sobre a vacinação, o que não impediu a atuação ética dos profissionais, que atuavam com respeito aos pacientes, tentando de forma adequada, levar informações aos interessado pois em muitas ocasiões, os pacientes tinham interesse em repassar à amigos e familiares, orientações de fonte confiável.

Vale ressaltar que o estabelecimento dessa comunicação assertiva, principalmente por parte dos profissionais, é essencial para o sucesso da vacinação, visto que, um estreitamento da relação profissional da saúde - paciente, é essencial para que haja adesão às orientações repassadas (Chou \& Budenz, 2020).

Assunção e Queiros (2015) afirmam que a relação profissional de saúde-paciente é construída por meio da comunicação, verbal e não verbal, estabelecida no contexto em que essa interação acontece, e como a qualidade dessa relação tem influência no tratamento e prevenção das doenças e na promoção da saúde, principalmente no que se refere à adesão de pacientes ao tratamento e à comunicação em saúde.

Para mais, durante a realização da campanha foi identificada a dificuldade na prática dos direitos e deveres do públicoalvo a ser vacinado e das equipes de vacinação. Nesse contexto, pode-se citar a dificuldade na comunicação com os usuários ou com seus acompanhantes, apesar disso os profissionais cumpriram o dever em "atender e orientar os usuários com segurança, responsabilidade e respeito" (Brasil, 2014).

Cita-se também a dificuldade por parte de algumas pessoas em compreender o direito dos membros da equipe de vacinação de preservar a sua imagem e não serem reproduzidos mídias com a sua imagem sem sua autorização, previsto no artigo 21 da Resolução COFEN n 564/2017, no qual o profissional pode "negar-se a ser filmado, fotografado e exposto em mídias sociais durante o desempenho de suas atividades profissionais".

\section{Conclusão}

A pandemia da COVID-19 enfatizou a problemática da propagação de informações falsas e negacionismo diante de um determinado cenário, tornando os indivíduos cada vez mais incrédulos em relação à ciência, políticas públicas e as principais medidas de prevenção e controle do vírus.

Foi possível encontrar os diferentes desafios durante a campanha de vacinação contra a Covid-19, entre eles: a organização logística do espaço físico para o condicionamento das pessoas, o acolhimento e sua humanização, assim como, suas subdivisões e a interação bilateral entre profissionais e os futuros vacinados e seus familiares. Além disso, apontou a propagação de informações falsas sobre o imunobiológico, aspecto que apresentou bastante impacto na adesão e confiabilidade dos idosos.

Os desafios encontrados enfatizaram a necessidade do fortalecimento das políticas públicas e promoção de informações corretas, com embasamento científico e maior valorização da equipe responsável pela imunização, pautada no respeito e boas condições de trabalho. Ressalta-se ainda que vivências como essa, apresentam impacto na formação profissional e pessoal dos participantes, que se depararam com diferentes situações e problemáticas, necessitando maior capacidade de adaptação, aplicação de conhecimento científico associada a prática, trabalho em equipe e humanização durante todo o processo de vacinação.

Diante dos achados nesse estudo, verifica-se a necessidade de um maior aprofundamento sobre a temática com a adoção de novas pesquisas sobre o atual cenário na tentativa de minimizar os problemas e consequentemente agilize e colabore com o processo de vacinação da população. Dessa forma, o número de vacinados na região se torna maior, reduzindo o número de 
contaminação e consequentemente as taxas de complicações e óbitos por COVID-19, principalmente entre os considerados grupos de risco, a exemplo dos idosos.

\section{Referências}

Atehortua, N. A., \& Patino, S. (2021). COVID-19, a tale of two pandemics: novel coronavirus and fake news messaging. Health Promot Int., $36(2): 524-534$.

Assunção, G. S., \& Queiroz, E. (2015). Abordagem do tema "Relação profissional de saúde-paciente" nos cursos de saúde da Universidade de Brasília. Psicologia: Ensino \& Formação. 6(2): 18-36.

Brasil. Ministério da Saúde. Secretaria de Vigilância em Saúde. Departamento de Vigilância das Doenças Transmissíveis. (2014). Manual de Normas e Procedimentos para Vacinação, 176 p.

Brasil. Ministério da Saúde. Secretaria de Vigilância em Saúde. Departamento de Imunizações e Doenças Transmissíveis. (2020). Manual de vigilância epidemiológica de eventos adversos pós-vacinação, 4. ed.

Brasil. Ministério da Saúde. Secretaria de Vigilância em Saúde. Departamento de Imunizações e Doenças Transmissíveis. (2021). Plano Nacional De Operacionalização Da Vacinação Contra A COVID-19, 6. ed.

Cavalcante, B. L. L., \& Lima, U. T. S. (2012). Relato de experiência de uma estudante de Enfermagem em um consultório especializado em tratamento de feridas. J Nurs Health, 1(2):94-103.

Chou, W. S., \& Budenz, A. (2020). Considering Emotion in COVID-19 Vaccine Communication: Addressing Vaccine Hesitancy and Fostering Vaccine Confidence. Health Commun, 35(14):1718-1722.

Hamada, R. A., Brasil, V., Souza, I., Campos, R., \& Leite, I. (2018). Conhecendo o Sistema Único de Saúde: um olhar da população. Revista de APS, 21.

Mendes, K. D. S., Silveira, R. C. C. P., \& Galvão, C. M. (2019). Uso de gerenciador de referências bibliográficas na seleção dos estudos primários em Revisão Integrativa. Texto \& Contexto Enfermagem, 28, e20170204.

Minayo, M. C. de S. (2012). Análise qualitativa: teoria, passos e fidedignidade. Ciência \& Saúde Coletiva, 17(3), 621-626.

Montezeli, J. H., \& Haddad, M. C. F. L. (2016). Habilidades sociais e à prática profissional em enfermagem. Ciência, Cuidado E Saúde, 15(1), 1-2.

OPAS. (2021). Histórico da pandemia de COVID-19. <https://www.paho.org/pt/covid19/historico-da-pandemia-covid-19>.

Perniciotti, P., Serrano Júnior, C. V., Guarita, R. V., Morales, R. J., \& Romano, B. W. (2020). Síndrome de Burnout nos profissionais de saúde: atualização sobre definições, fatores de risco e estratégias de prevenção. Revista da SBPH, 23(1), 35-52.

Resolução COFEN nº 564/2017, de 6 de novembro de 2017 (2017). Dispõe sobre a aprovação do novo Código de Ética dos Profissionais de Enfermagem. Brasília, DF.

Ribeiro, L. M., Vieira, T. A. \& Naka, K. S. (2020). Síndrome de burnout em profissionais de saúde antes e durante a pandemia da COVID-19. Revista Eletrônica Acervo Saúde, 12(11), e5021.

Sato, A. P. S. (2018). What is the importance of vaccine hesitancy in the drop of vaccination coverage in Brazil? Revista De Saúde Pública, 52, 96.

Santos, E. P. (2017). Guia de boas práticas de imunização em áreas remotas de difícil acesso. SBIM, 1, 1-44.

SESPA. (2021). Plano Paraense De Vacinação -PPV/COVID-19. <http://www.saude.pa.gov.br/wp-content/uploads/2021/01/PLANO_PARAENSE_ DE_VACINACAO_FINAL-1.pdf>.

Vilanova, M. (2020). Vacinas e imunidade, Rev. Ciência Elem., 8(2):021. 